DOE/NV/11630

UC-703

\title{
Advanced Radiation Detector Development: Advanced Semiconductor Detector Development: Development of a Room-Temperature, Gamma Ray Detector Using Gallium Arsenide to Develop an Electrode Detector
}

\author{
prepared by \\ RECEIVED \\ NOV 211995 \\ Glenn F. Knoll \\ Department of Nuclear Engineering \\ OSTI \\ College of Engineering \\ The University of Michigan \\ Ann Arbor, Michigan 48109
}

Publication No. 1

submitted to

Nevada Operations Offfice

U.S. Department of Energy

Las Vagas, Nevada

November 1995

The work upon which this report is based is supported by the U.S.

Department of Energy under Grant \# DE-FG08-94NV11630. 
This report was prepared as an account of work sponsored by the United States Government. Neither the United States nor the United States Department of Energy, nor any of their employees, makes any warranty, express or implied, or assumes any legal liability or responsibility for the accuracy, completeness or usefulness of any information, apparatus, product or process disclosed, or represents that its use would not infringe privately owned rights. Reference herein to any specific commercial product, process, or service by trade name, mark, manufacturer, or otherwise, does not necessarily constitute or imply its endorsement, recommendation, or favoring by the United States Government or any agency thereof. The views and opinions of authors expressed herein do not necessarily state or reflect those of the United States Government or any agency thereof.

This report has been reproduced directly from the best available copy.

Available to DOE and DOE contractors from the Office of Scientific and Technical Information, P.O. Box 62, Oak Ridge, TN 37831; prices available from (615) 576-8401.

Available to the public from the National Technical Information Service, U.S. Department of Commerce, 5285 Port Royal Rd., Springfield, VA 22161. (703) $487-4650$ 


\section{DISCLAIMER}

Portions of this document may be illegible electronic image products. Images are produced from the best available original document. 


\section{ANNUAL PROGRESS REPORT: Advanced Radiation Detector Development \\ U.S. DEPARTMENT OF ENERGY - LAS VEGAS OPERATIONS OFFICE \\ September 30,1994 - SEPTEMBER29, 1995 \\ Submitted by Glenn F. Knoll \\ University of Michigan}

\section{INTRODUCTION AND OVERVIEW}

The advanced detector development project at the University of Michigan has completed the first full year of its current funding. Our general goals are the development of

radiation detectors and spectrometers that are capable of portable room temperature operation.

Over the past 12 months, we have worked primarily in the development of semiconductor spectrometers with "single carrier" response that offer the promise of room temperature operation and good energy resolution in gamma ray spectroscopy. We have also begun a small scale effort at investigating the properties of a small non-spectroscopic detector system with directional characteristics that will allow identification of the approximate direction in which gamma rays are incident. These activities have made use of the extensive clean room facilities at the University of Michigan for semiconductor device fabrication, and also the radiation measurement capabilities provided in our laboratory in the Phoenix Building on the North Campus.

In addition to our laboratory based activities, Professor Knoll has also been a participant in several Department of Energy review activities held in the Forrestal Building and at the Germantown site. The most recent of these has been service on a DOE review panel chaired by Dr. Hap Lamonds that is reviewing the detector development programs supported through the Office of Arms Control and International Security.

\section{TECHNICAL PROGRESS}

A. Investigation of a three electrode semiconductor detector with internal grid

In many compound semiconductor materials that are candidates for room temperature detectors, there is a problem with the mobility of the holes that carry the positive charges created along the particle track. This poor hole mobility leads to incomplete charge collection 
that consequently limits the energy resolution achievable in a conventional detector structure. In many of these materials, the electron mobility is much higher and, therefore, the electron collection tends to be much more complete.

This situation is entirely analogous to the charges created by ionization in a gas. The electron mobility in a gas is orders of magnitude higher than that of the positive ions which carry the positive electrical charge. A classic solution to this problem in the case of ion chambers dates back many decades and involves the use of a "Frisch grid" inserted between the anode and cathode that surround the active volume of the gas. Electrons are drawn toward the grid, pass through it, and a signal is developed by their motion only between the grid and anode. If the grid/anode spacing is made much smaller than the grid/cathode distance, than the majority of all gamma ray interactions will take place between the grid and cathode. For all such events, the electrons travel the same distance between the grid and anode and, therefore, contribute the same incremental induced charge to the pulse that is developed. The positive ions travel in the opposite direction and to first order, do not contribute to the output signal. Thus, a pulse is developed that is dependent only on the motion of the electrons and not on the motion of the positive ions.

The same principle should be applicable in semiconductor detectors. However, it is much more difficult to fabricate an internal grid between the two contacts on the surfaces of a semiconductor wafer. We have set about to do so using silicon because of its well developed processing technology. Our intent is to investigate the feasibility of fabricating a three electrode device in silicon, and, if successful, to demonstrate a proof-of-principle that single carrier response can be obtained. Once we have investigated the properties of this approach in silicon, we will then plan to extend the fabrication technology to compound semiconductor materials where hole transport is much more of a problem.

To date, we have gone through two fabrication cycles of these three-electrode devices. Our basic approach is to use ion implantation to create a grid pattern on the surface of a high resistivity silicon wafer, to create a grid to anode space by growth of an epitaxial layer on top 
of the grid, and then to etch selectively to provide electrical contacts to the grid at the edges of the wafer. Our first batch using these techniques resulted in excessive leakage currents due to inadequately passivated surfaces. We, therefore, revised our processing steps and a new procedure was described in our progress report of several months ago, and will not be repeated here. We have just completed the processing steps using this new technique on a set of wafers, and are now in the process of mounting them to allow measurement of their characteristics. Technical details are given in Appendix I of this report.

\section{B. Coplanar electrode structures on cadmium-zinc-telluride}

We have also been carrying out studies of the behavior of cadmium-zinc-telluride (CZT) fitted with a coplanar electrode structure similar to that first described by Paul Luke at the 1994 IEEE Nuclear Science Symposium. We have obtained excellent results to date, and are very enthusiastic about this approach. We have included several design innovations that are different from those of other groups investigating this technique. First, we have chosen an electrode pattern that allows only two wires to emerge from the detector package, as opposed to individual wires for each strip used by other groups. This approach greatly simplifies the bonding requirements and leads to a more rugged configuration. We are also studying the effects that asymmetries in these electrode patterns have on the detailed performance of the detectors. We have recently obtained a two dimensional electrostatic modeling code that has revealed some interesting features of the internal electric field structure near the surface of the coplanar strips. We feel that we are beginning to understand some of the limitations that are being observed in current devices, and are now pursuing ways to improve the energy resolution through more advanced designs.

We have also been the first group to investigate the use of induced signals at the cathodes to provide information on the depth of the interaction in the interior of the detector. By varying the pulse shaping time of the amplifiers used to process the cathode signal, we are able to obtain a pulse amplitude that depends primarily on only the electron motion, and hence is linearly proportional to the distance travelled by electrons from the interaction position to the 
anode. We are now beginning to use this added information to allow correction for the small amount of electron trapping that exists in the bulk of the material, and are hopeful that significant improvements in energy resolution will result.

These developments were the subject of a paper presented at the Room-Temperature Semiconductor Detector Conference recently held in Grenoble, and a copy of a paper presented at that conference by our group is included as Appendix II.

\section{Directional gamma ray detector}

There are circumstances in which being able to provide some rough information regarding the general direction from which gamma rays are incident on a detector would be of interest. It would allow the determination of whether a source might be to the left or right of the observer, to the front or back, and its localization to within perhaps twenty to thirty degrees angular resolution. While this performance would be far from an imaging capability, it could permit the association of a given source with known structures or other objects within the field of view.

A graduate student on the project is therefore undertaking a modeling study of a suggested configuration for this type of device. It relies on the fact that when incident gamma rays interact with an absorber, fluorescent x-rays are emitted by that material following photoelectric absorption. These fluorescent $x$-rays have short ranges and can be easily shielded. We have begun modeling a proposed detector configuration in which a cube of lead is fitted with thin CZT detectors that are "buried" several millimeters below the surface of each face of the cube. These detectors are relatively transparent to gamma rays above several hundred $\mathrm{keV}$, but will be thick enough to intercept the $80-90 \mathrm{keV}$ fluorescent $\mathrm{x}$-rays emitted from lead. We are applying some modeling tools that have been developed over the past 10 years in the Department of Nuclear Engineering to predict the directional capabilities of such a device and also to optimize its design. Further details are given in Appendix III. 


\section{APPENDIX I}




\section{SINGLE CARRIER DEVICE - BURIED STRUCTURE.}

\section{RON ROJESKI and BISHNU GOGOI}

The following describes the status of the fabrication of a single carrier device using a buried grid structure in a silicon substrate. The device was fabricated using high resistivity $(8 \mathrm{~K} \mathrm{ohm}-\mathrm{cm}$ ) double-side polished silicon wafers. The fabrication process uses a 6 mask process. The final device is mounted on a boron-nitride package with five contact pins. The contact pins are as follows

1. Front $n+$ epitaxial layer contact

2. Front $p+$ grid contact

3. Front $\mathrm{p}++$ isolation ring contact

4. Backside $\mathrm{p}++$ substrate contact

5. Backside $\mathrm{p}++$ isolation ring contact

\section{DEVICE FABRICATION:}

\section{a) Process Flow:}

The fabrication of the device is briefly described here. The starting wafer is a high resistivity double polished float-zone silicon wafer. The processing steps with the corresponding mask are as follows.

1. Encapsulate the wafer with $3000 \mathrm{~A}$ of thermal oxide.

2. Pattern and etch alignment marks ( Mask \#1)

3. Pattern contact ring to the grid lines and perform $p++$ diffusion (Mask \#2)

4. Pattern gridlines and perform $p+$ implant ( Mask \#3)

5. Metal ( $\mathrm{Cr} / \mathrm{Au}$ ) evaporation and liftoff ( Same as Mask \#1)

6. Oxide pattern and etch using backside IR alignment and p++ implant (Mask \#4)

7. Strip top oxide and grow 30 microns of high resistivity n-epi with 1 micron of $n++$ epi on top.

8. Metal ( $\mathrm{Cr} / \mathrm{Au}$ ) evaporation front and back side; protect backside and pattern front side and etch metal. Use TMAH ( tetra methyl ammonium hydroxide) to etch epitaxial layer down to the $\mathrm{p}++$ contact ring. (Mask \#5)

9. Grow passivation oxide of $3000 \mathrm{~A}$ and pattern/etch contact holes to the front side and backside. Deposit metal $(\mathrm{Cr} / \mathrm{Au})$ and perform liftoff to define the contacts to the device. ( Mask \#6)

10. Spin resist on top of wafer and dice the samples. Soak samples in acetone. 
11. Mount device to specially designed boron nitride package with high resistivity epoxy that is doped with boron nitride.

12. Wire bond contact pads on device to terminals of package.

b) Discussion of critical process steps:

Some of the critical points in the fabrication of the device are discussed below :

1. The surface of the wafer must be thoroughly cleaned to prevent degradation of device performance.

2. The $\mathrm{p}+$ implant must be sufficently high so as to provide a sufficient etch stop during the etch back to the contact ring.

3. The TMAH etch process must be monitored very carefully since the etch rate is very temperature dependent. This procedure is a very well timed etch.

4. The patterning of the contact holes on the front side after the 30 micron TMAH etch back is done using a special thick resist. The very high steps on the wafer surface cannot be adequately covered using conventional resist.

5. The patterning of the contact holes on the back side of the wafer is done using a special technique. Since the front surface is highly irregular due to the TMAH etchback, the wafer cannot be spun with resist as with a normal wafer. The chuck for the resist spinner as well as the mask aligner cannot generate enough vacuum to hold the wafer. Therefore the wafer is mounted on a smooth wafer and the lithography is performed.

\section{DEVICE TESTING:}

Some electrical testing of the device has been performed. The pn diode between the $\mathrm{p}+$ grid and the $\mathrm{n}+$ epi layer was tested. The forward characteristic showed a turn-on voltage of 0.6 volts while the reverse bias characteristic showed a leakage current 74 microAmps at a reverse bias voltage of -1 Volt. The pn junction between the backside p++ substrate and the $p+$ grid was floating. It is expected that if this junction is kept reversebiased, the reverse leakage current will be reduced.

Electrical testing of the mounted device is under way. Testing with radiation sources will be performed when the electrical testing is completed. 


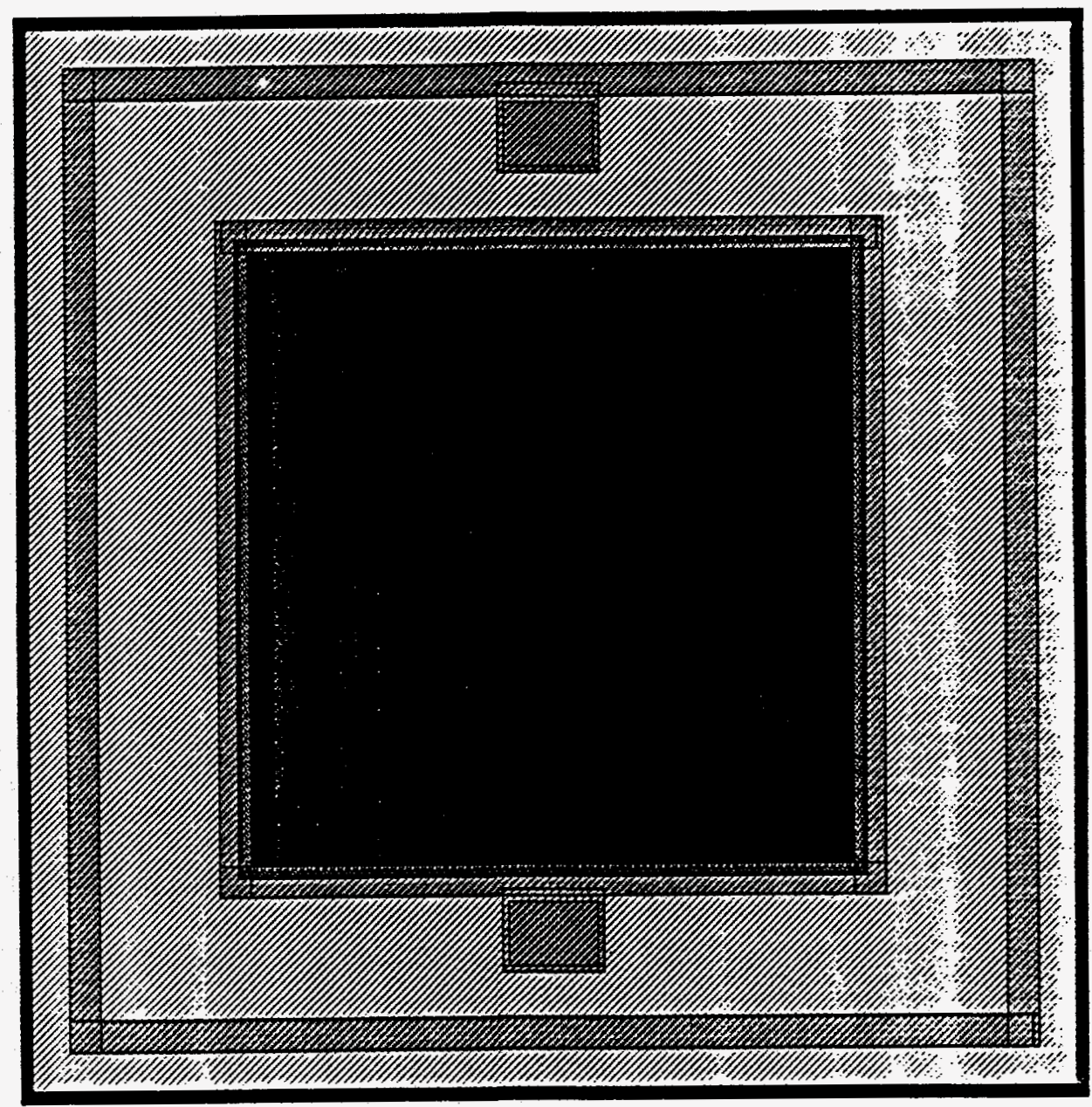

LAYOUT OF COMPLETE DEVICE 


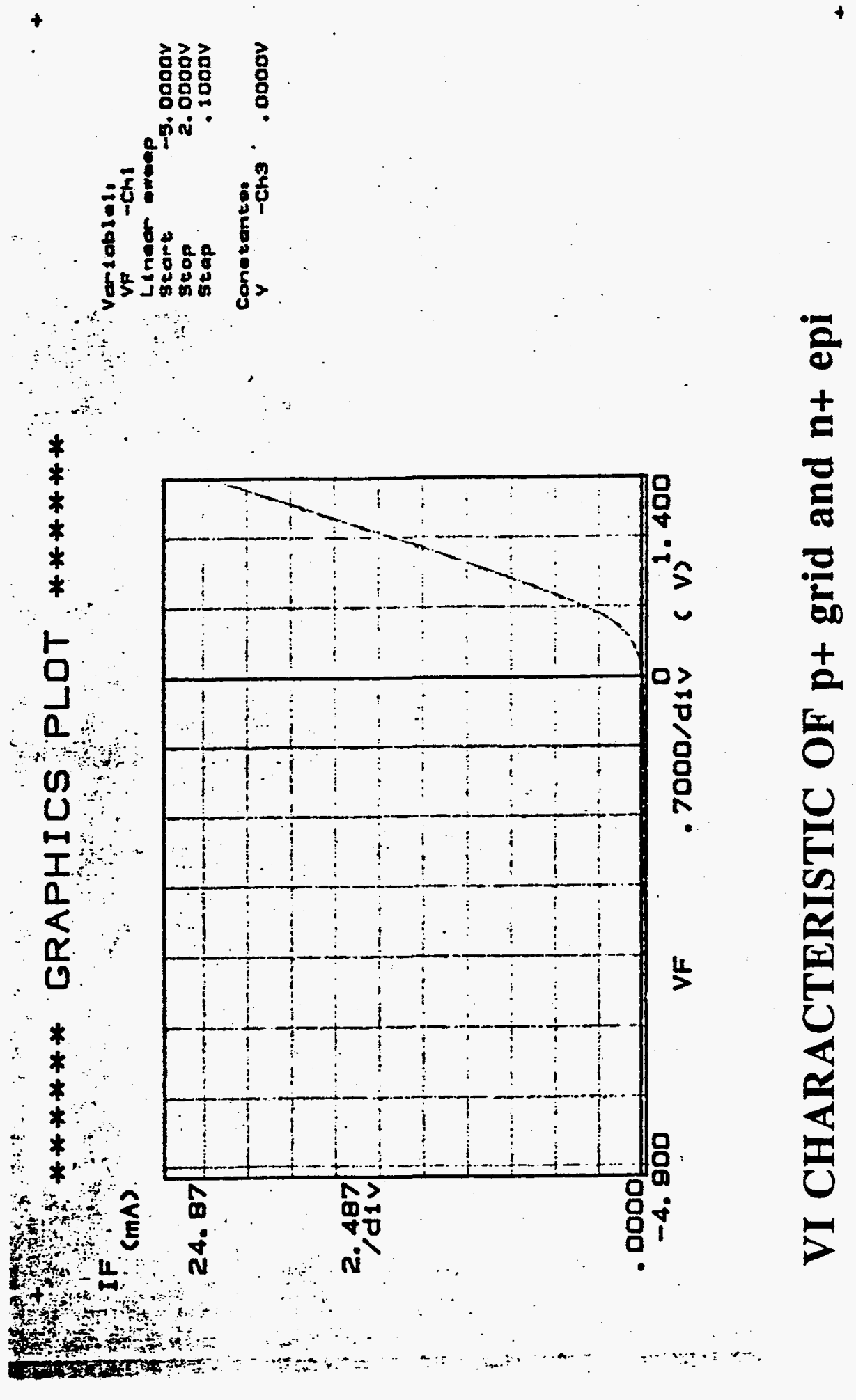


APPENDIX II 


\title{
1-D Position Sensitive Single Carrier Semiconductor Detectors
}

\author{
Zhong He, Glenn F. Knoll, David K. Wehe, Ronald Rojeski, Carlos H Mastrangelo*, \\ Mark Hammig, Carla Barrett, Akira Uritani \\ Department of Nuclear Engineering \\ *Department of Electrical Engineering and Computer Engineering \\ University of Michigan \\ Ann Arbor, MI, USA 48109-2104
}

\begin{abstract}
A single polarity charge sensing method has been studied using coplanar electrodes on $5 \mathrm{~mm}$ cubes of $\mathrm{CdZnTe} \gamma$-ray detectors. This method can ameliorate the hole trapping problem of room-temperature semiconductor detectors. Our experimental results confirm that the energy resolution is dramatically improved compared with that obtained using the conventional read out method, but is still about an order of magnitude worse than the theoretical limit.

A method to obtain $\gamma$-ray interaction depth between the cathode and the anode is presented here. This technique could be used to correct for the electron trapping as a function of distance from the coplanar electrodes. Experimental results showed that a position resolution of about $0.9 \mathrm{~mm}$ FWHM at $122 \mathrm{keV}$ can be obtained. These results will be of interest in the design of higher performance room-temperature semiconductor $\gamma$-ray detectors.
\end{abstract}




\section{Introduction}

Room-temperature semiconductor $\gamma$-ray detectors having high atomic numbers and wide band gaps have long been under development. Among those, $\mathrm{HgI}_{2}[1,2], \mathrm{CdTe}[3]$ and CdZnTe [4] detectors have shown the most promise. Important progress on detector performance has been achieved through improving the material quality $[4,5]$ and applying different signal processing methods $[6,7]$. Nevertheless, the energy resolution achieved using conventional planar electrodes is still far from the theoretical expectation. Charge trapping [4] and polarization [8] effects are the dominant problems which have limited the energy resolution. Recently, Luke $[9,10]$ employed a method analogous to the Frisch grids [11] commonly employed in gas ion chambers, but using parallel coplanar strip electrodes. The strip electrodes on Luke's device were connected to give two sets of inter-digital grid electrodes. When charge carriers move within the bulk material of the detector, they induce equal amount of charges on both electrodes except when they are within a very short distance from the coplanar electrodes. By reading the difference signal between these two sets of electrodes, a net signal is only induced when the charge carriers are moving in close proximity to the coplanar electrodes. When electron-hole pairs are generated by $\gamma$-ray interaction with the detector material, only one type of charge carriers (electron or hole) will move towards the coplanar electrodes. As a result, single polarity charge sensing can be obtained. For commonly used semiconductor detectors, signals from electrons are chosen so that the hole trapping problem can be eliminated. Using this approach, Luke found a significant improvement in the energy resolution of a $5 \mathrm{~mm}$ cube CdZnTe detector - Following Luke's work, He [12] derived the analytical approximation of the electric field distribution within semiconductor detectors for more generalized configurations of coplanar strip electrodes.

In this work, we propose a method to make a semiconductor detector with coplanar electrodes position sensitive to the $\gamma$-ray interaction depth between the cathode and coplanar anodes. This technique will provide an important capability for room-temperature semiconductor detectors in $\gamma$-ray astronomy, medical imaging and other industrial applications. Furthermore, since the energy spectra can be obtained as a function of the interaction depth, electron trapping can be monitored at different distances from the anode. This would provide a more accurate correction for electron trapping than the linear compensation method [10].

\section{Principle}

The basic structure of coplanar strip electrodes used by Luke [9] is shown schematically in Fig. 1 , where $\mathrm{V}_{d}$ is the detector bias voltage, $\mathrm{V}_{g}$ is the grid bias voltage and $\mathrm{P}$ is the spatial period of the strip electrodes. Since electron sensing is preferred, a negative detector bias voltage was used so that electrons move towards the coplanar anodes. The associated electronics are shown schematically in Fig. 2. The induced signals on the two coplanar electrodes are read out using two AC-coupled conventional charge sensitive preamplifiers. The signal subtraction circuit consists of only one operational amplifier and 
four resistors, one of them can be adjusted in order to change the relative gain of the two signal channels. From Ramo-Shockley theorem $[13,14]$ and previous analysis $[10,12]$, the induced charge on the collecting anode as a function of time is illustrated in Fig. 3(a). For simplicity, it was assumed that there is no electron or hole trapping. One can see that the relative amplitudes of the signal induced by the electron motion are $0.5,0.75$ and 1.0 when the $\gamma$-ray interaction locations are at the anode, middle of detector, and the cathode, respectively. Since the electron induced pulse has a much faster rise time than that of the holes, the pulse amplitude (out2) due solely to the electron motion can be measured by using a shaping amplifier with a shaping time constant which is long compared to the rise time of electron component, but short compared to that induced by hole motion. On the other hand, the pulse amplitude (out1) of the subtraction circuit is always proportional to the number of electrons that reach the coplanar electrodes. Therefore, the ratio of the two output amplitudes out2/out1 should be linearly proportional to the distance of the $\gamma$-ray interaction from the anodes. In practice, there is always some hole signal mixed in with the electron component, and the number of electrons arriving at the coplanar anodes decreases non-linearly as the interaction distance increases from the anodes. Therefore, the ratio value as a function of interaction depth will show some non-linear effect. However, this ratio value will increase monotonically as the distance increases from the coplanar anodes.

The induced charge on the cathode as a function of time is illustrated in Fig. 3(b). Using the same principle suggested above, position sensing can also be achieved by dividing the signal obtained from a preamplifier AC coupled to the cathode and shaped using a short shaping time constant by that from the coplanar electrodes.

\section{Results at $122 \mathrm{keV}$}

The technique suggested above has been tested using a $5 \mathrm{~mm}$ cube spectrometer grade $\mathrm{CdZnTe}$ detector (No.720109Co). The crystal and the electrodes were manufactured and processed by DIGIRAD. The experimental setup is schematically shown in Fig. 4(a). Three slots in the $2 \mathrm{~cm}$ thick $\mathrm{Pb}$ collimator have the same width of $0.5 \mathrm{~mm}$ and are separated by $1 \mathrm{~mm}$ thick $\mathrm{Pb}$, and served to provide narrow beams of $\gamma$-rays at fixed depths. The center to center distance of neighboring $\gamma$-ray beams was thus $1.5 \mathrm{~mm}$. Fig. 4(b) plots the counts as a function of observed signal ratio for the 3 beam irradiation. Note that the adjacent peaks are roughly equal separated along the ratio axis, although a non-linear effect is evident. A position resolution of about $0.9 \mathrm{~mm}$ was obtained, without correction for the width of the slots. During the measurements, the detector bias was -700 volts and the grid bias was +15 volts. Both shaping time-constants for the collecting electrode signal and the coplanar electrodes signal were $2 \mu \mathrm{s}$. Since the rise time of the hole component is about $10 \mu \mathrm{s}$, the $2 \mu$ s shaping time is still short for hole signals.

In another experiment, the ${ }^{57}$ Co source was located about $1 \mathrm{~cm}$ above the top of the detector to provide a uniform incident $\gamma$-ray field between the cathode and the anodes. The energy spectra as a function of ratio value is plotted in Fig. 5(a). The photo-peak amplitude of $122 \mathrm{keV} \gamma$-rays as a function of the ratio, which is approximately proportional 
to the interaction distance from the anode, is shown in contrast in Fig. 5(b). The gains of the collecting electrode and coplanar electrodes signals were set equal so that no electron trapping correction was made. The energy resolution decreased from $\sim 24 \%$ to $\sim 11 \%$ FWHM when the $\gamma$-ray interaction position moved from the coplanar electrodes to the cathode. One can also see that the amplitude decreased slowly as the distance from the anode increased in the central part of the detector, and changes more rapidly when it approaches either end. It deviates from the exponential decrease expected for uniform electron trapping within the detector volume. This indicates that other factors, in addition to the electron trapping, affect the amplitude of the signals. Further investigation is on going.

\section{Results at $662 \mathrm{keV}$}

Experiments were also carried out using a different $5 \mathrm{~mm}$ cube $\mathrm{CdZnTe}$ detector (No.710008T) with electrodes patterned by DIGIRAD. This CdZnTe crystal was obtained from eV Products. The energy spectrum obtained using the coplanar electrodes is compared with that using the conventional planar method from $662 \mathrm{keV} \gamma$-rays in Fig. 6 . Note that the energy resolution is dramatically improved using the coplanar electrodes readout. The shaping time constant in both cases was $2 \mu \mathrm{s}$. The relative gain between the collecting and the non-collecting electrode signals was set to be equal by adjusting the resistance of the potentiometer in the subtraction circuit. Therefore, we did not compensate for electron trapping during this measurement. From Fig. 6, one can see that the energy resolution would be further improved if the shoulders at both sides of the photo-peak could be reduced.

In order to investigate the origination of the shoulders, we obtained energy spectra at different $\gamma$-ray interaction depths between the anodes and the cathode. Instead of using the ratio of the signals from the collecting electrode and the coplanar electrodes, we read out the electron motion signal from the cathode, and then divided by the signal obtained from the coplanar anodes. The disadvantage of this method is that it requires three charge sensitive pre-amplifiers, instead of the two needed by Luke's original circuit. But we chose this technique because the position sensitivity is less affected by the nonsymmetric electrode pattern on the coplanar anodes, and may yield better resolution due to the lower noise from the cathode compared to the collecting anode.

The energy spectra at $662 \mathrm{keV}$ as a function of interaction depth is plotted in Fig. 7. It is evident that the peaks were broadened by those $\gamma$-ray events which interacted in the detector half close to the anodes. Excellent energy resolution was obtained from the events near the cathode. When we grouped the energy spectra with a finer ratio step, an energy resolution approaching $2 \%$ FWHM was obtained near the cathode. Near the anode, the photo-peak amplitude could not even be obtained due to the degraded energy resolution. 


\section{Summary}

We confirmed that the significant improvement on the energy resolution can be achieved using coplanar electrodes readout technique suggested by Luke [9]. The position sensing technique presented in this paper will provide an important capability for roomtemperature semiconductor detectors, and will be a useful tool for investigating factors that affect the performance of these detectors. Comparison of the two position sensing techniques, which use signals from the collecting electrode and the cathode, is on going. The mechanisms which deteriorate the energy resolution when the $\gamma$-rays interact close to the anode need further investigation.

\section{Acknowledgments}

This work was supported by the Department of Energy under contract number DE-FG0894 NV11630.

\section{References}

[1] D. Ortendahl et al., "Operating characteristics of small position-sensitive mercuric iodide detectors", IEEE Transactions on Nuclear Science, vol. 29(1), pp. 784-788, 1982.

[2] B.E. Patt et al., "Mercuric iodide x-ray camera", IEEE Transactions on Nuclear Science, vol. 33(1), pp. 523-526, 1986.

[3] E. Raiskin and J.F. Butler, "CdTe low level $\gamma$ detectors based on a new crystal growth method", IEEE Transactions on Nuclear Science, vol. 35(1), pp. 81-84, 1988.

[4] J.F. Butler et al., "Cdznte gamma ray detectors", IEEE Transactions on Nuclear Science, vol. 39(4), pp. 605-609, 1992.

[5] L.V.D. Berg, "Recent developments in mercuric iodide technology at EG\&G Energy Measurements, Inc., Santa Barbara Operations", Nuclear Instruments and Methods in Physics Research, vol. A322, pp. 453-456, 1992.

[6] L.T. Jones and P.B. Woollam, "Resolution improvement in CdTe $\gamma$ detectors using pulse-shape discrimination", Nuclear Instruments and Methods, vol. 124, pp. 591$595,1975$.

[7] M. Richter and P. Siffert, "High resolution $\gamma$ ray spectroscopy with CdTe detector systems", Nuclear Instruments and Methods in Physics Research, vol. A322, pp. 529-537, 1992. 
[8] V. Gerrish, "Polarization and gain in mercuric iodide $\gamma$-ray spectrometers", Nuclear Instruments and Methods in Physics Research, vol. A322, pp. 402-413, 1992.

[9] P.N. Luke, "Single-polarity charge sensing in ionization detectors using coplanar electrodes", Appl. Phys. Lett., vol. 65(22), pp. 2884-2886, 1994.

[10] P.N. Luke, "Unipolar charge sensing with coplanar electrodes - application to semiconductor detectors", IEEE Transactions on Nuclear Science, vol. 42(4), pp. 207$213,1995$.

[11] O. Frisch, ", British Atomic Energy Report BR-49, 1944.

[12] Zhong He, "Potential distribution within semiconductor detectors using coplanar electrodes", to appear in Nuclear Instruments and Methods, 1995.

[13] S. Ramo, "Currents induced by electron motion", Proc. of the I.R.E., vol. 27(9), pp. $584,1939$.

[14] W. Shockley, "Currents to conductors induced by a moving point charge", Journal of Applied Physics, vol. 9, pp. 635-636, 1938.

\section{Figure Captions}

Fig.1: End-on view of the coplanar strip electrodes.

Fig.2: Schematic diagram of the electronic circuit.

Fig.3: Pulse shape induced on (a) collecting electrode and (b) cathode, from interactions (1) near coplanar anodes, (2) at detector mid-plane, (3) near cathode.

Fig.4: Position sensitivity measurement.

(a) Side view of experiment. (b) Counts versus output ratio.

Fig.5: (a) Energy spectra and (b) photo-peak amplitude vs interaction distance at $122 \mathrm{keV}$.

Fig.6: Comparison of spectrum using (a) conventional and (b) coplanar electrodes readout.

Fig.7: Energy spectra versus interaction distance at $662 \mathrm{keV}$. 
Collecting Electrode

Non-collecting Electrode

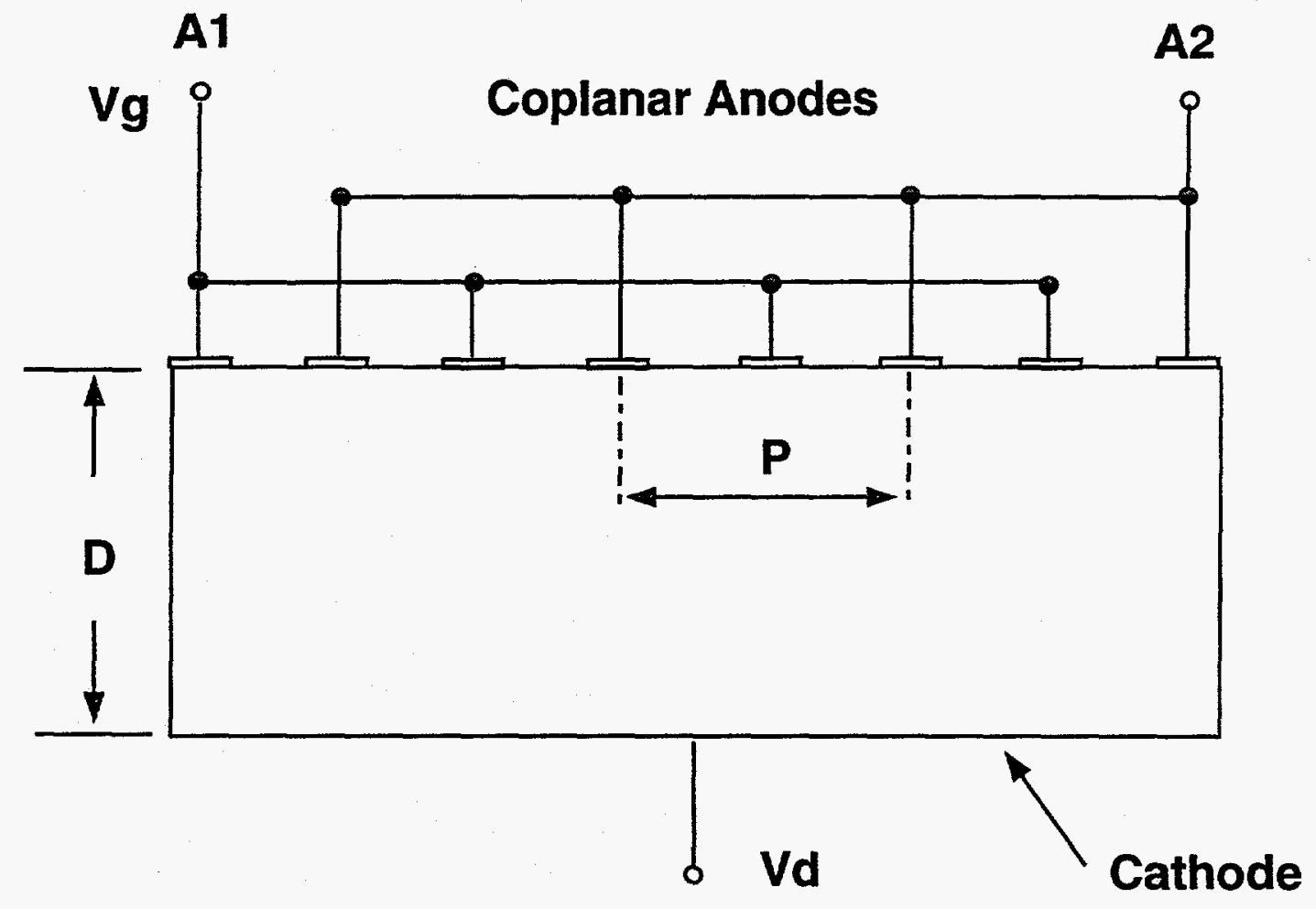

Fig.1 


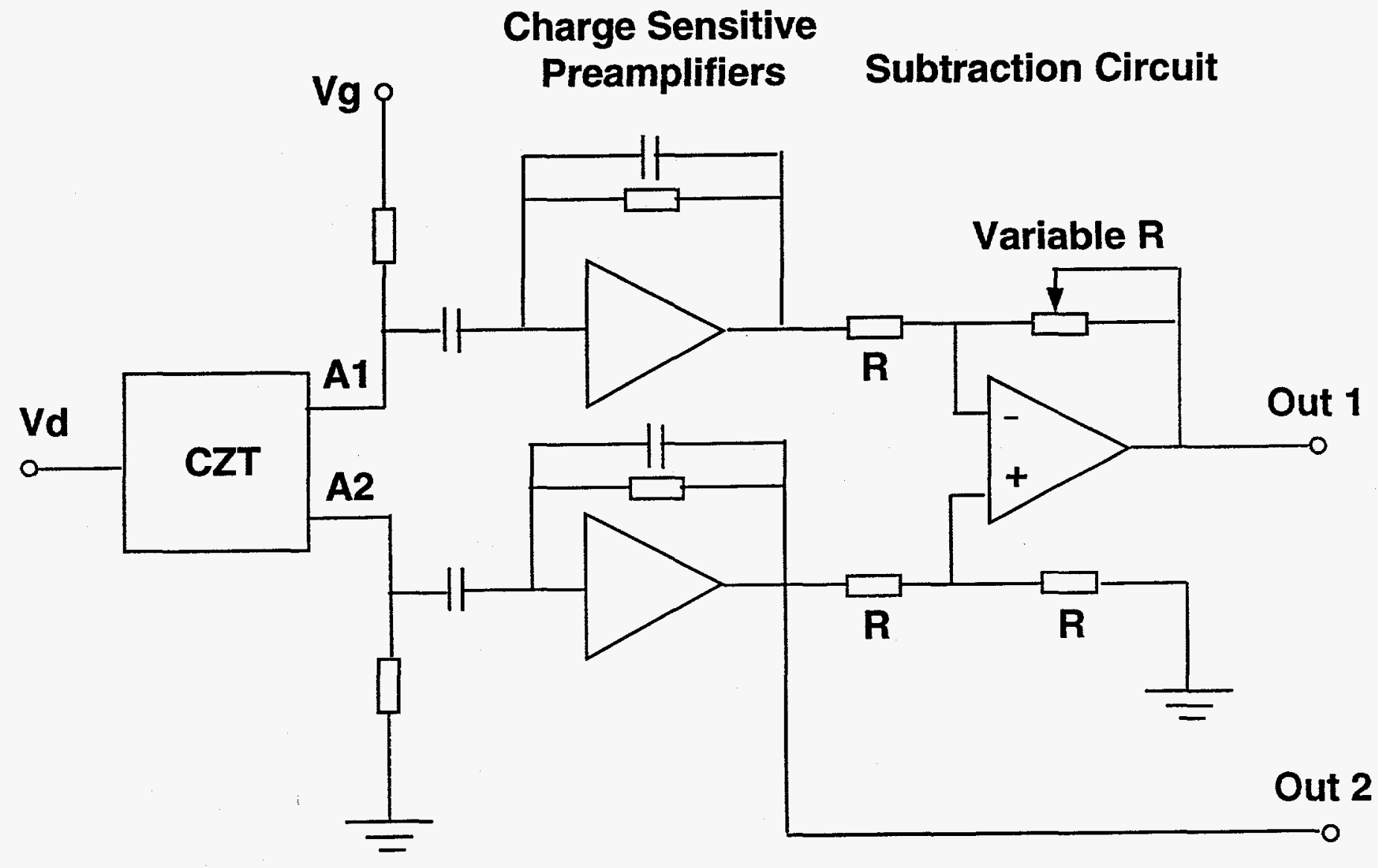

Fig.2 

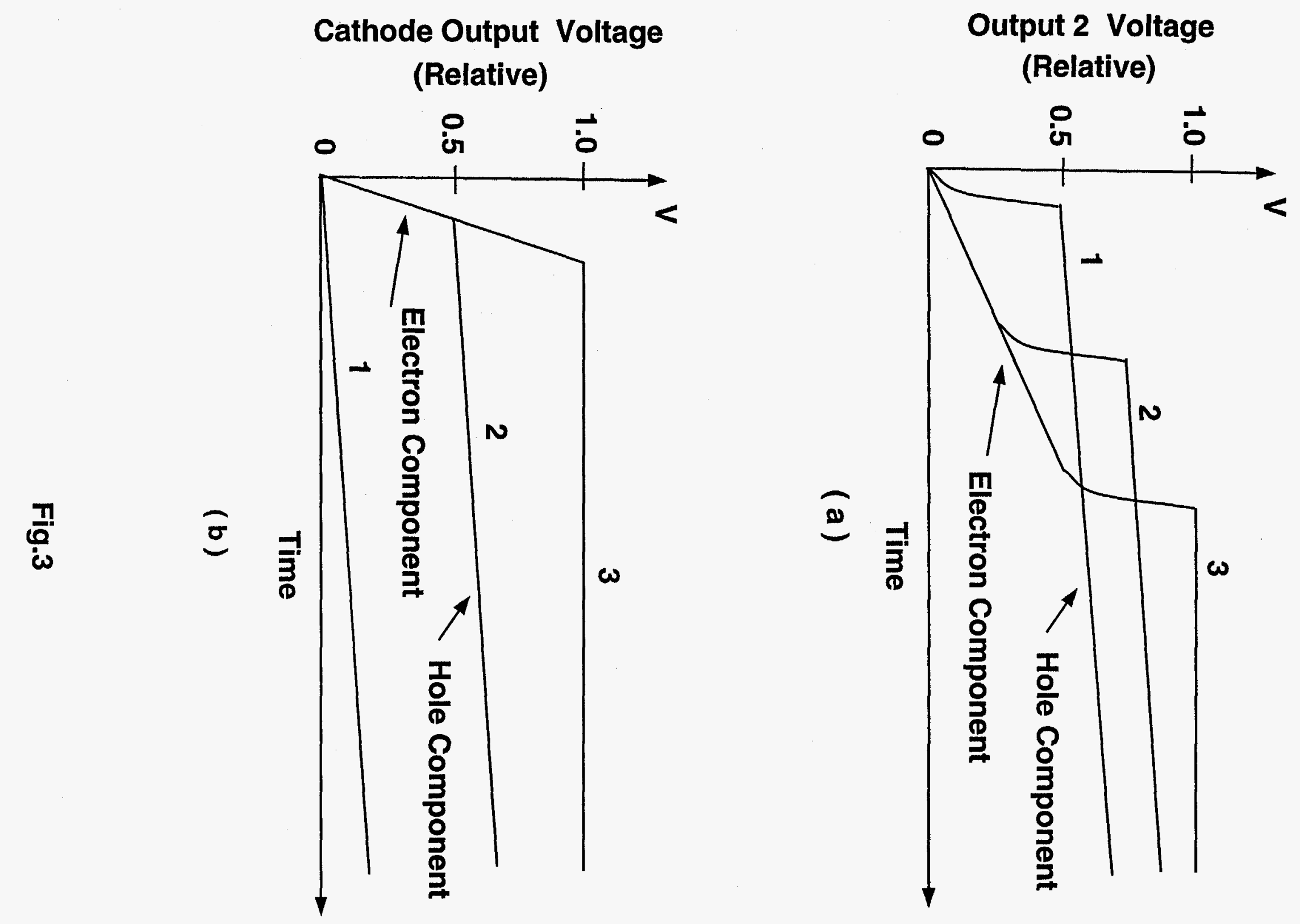
122 keV Gamma-Rays

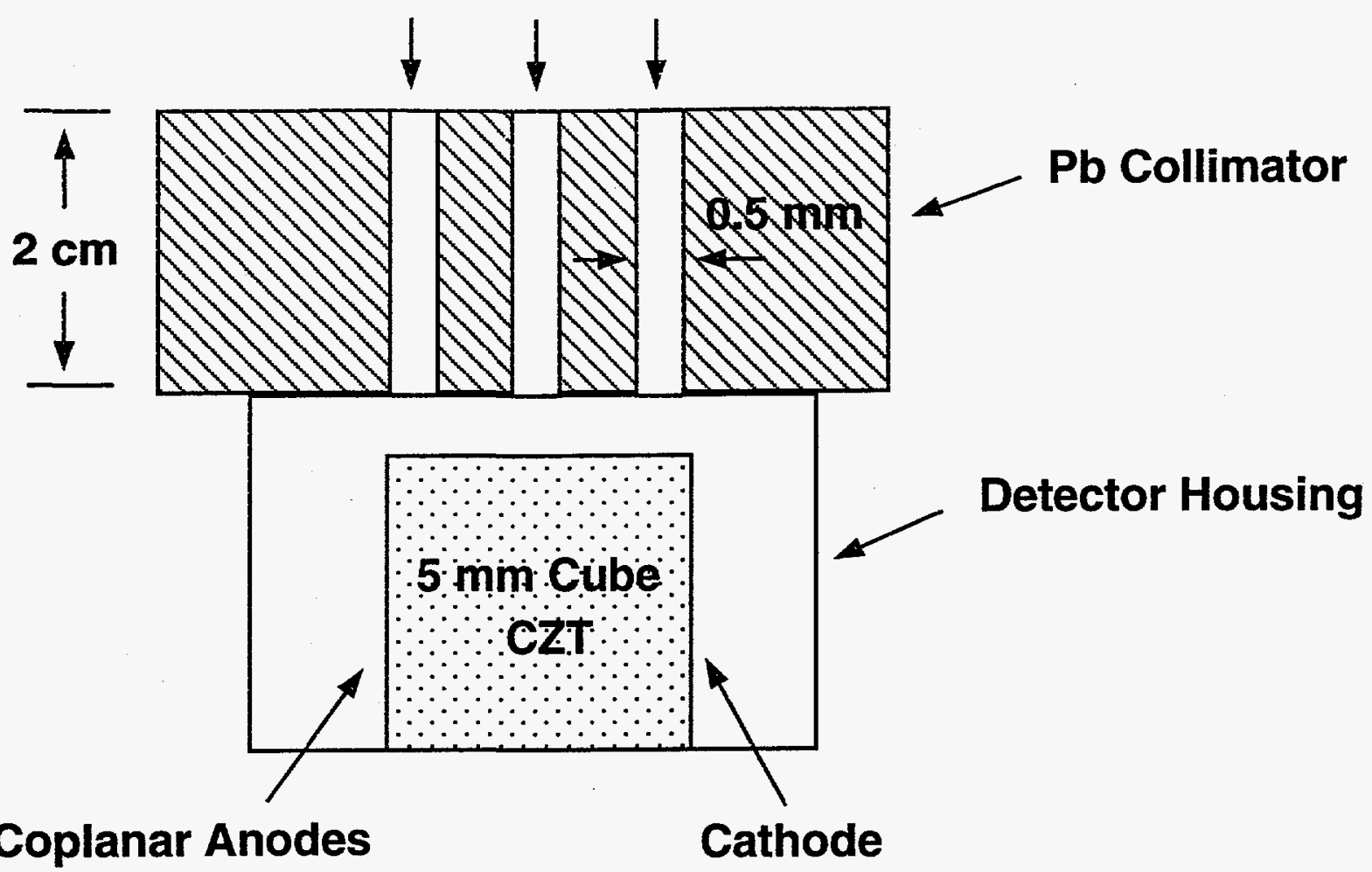

(a)

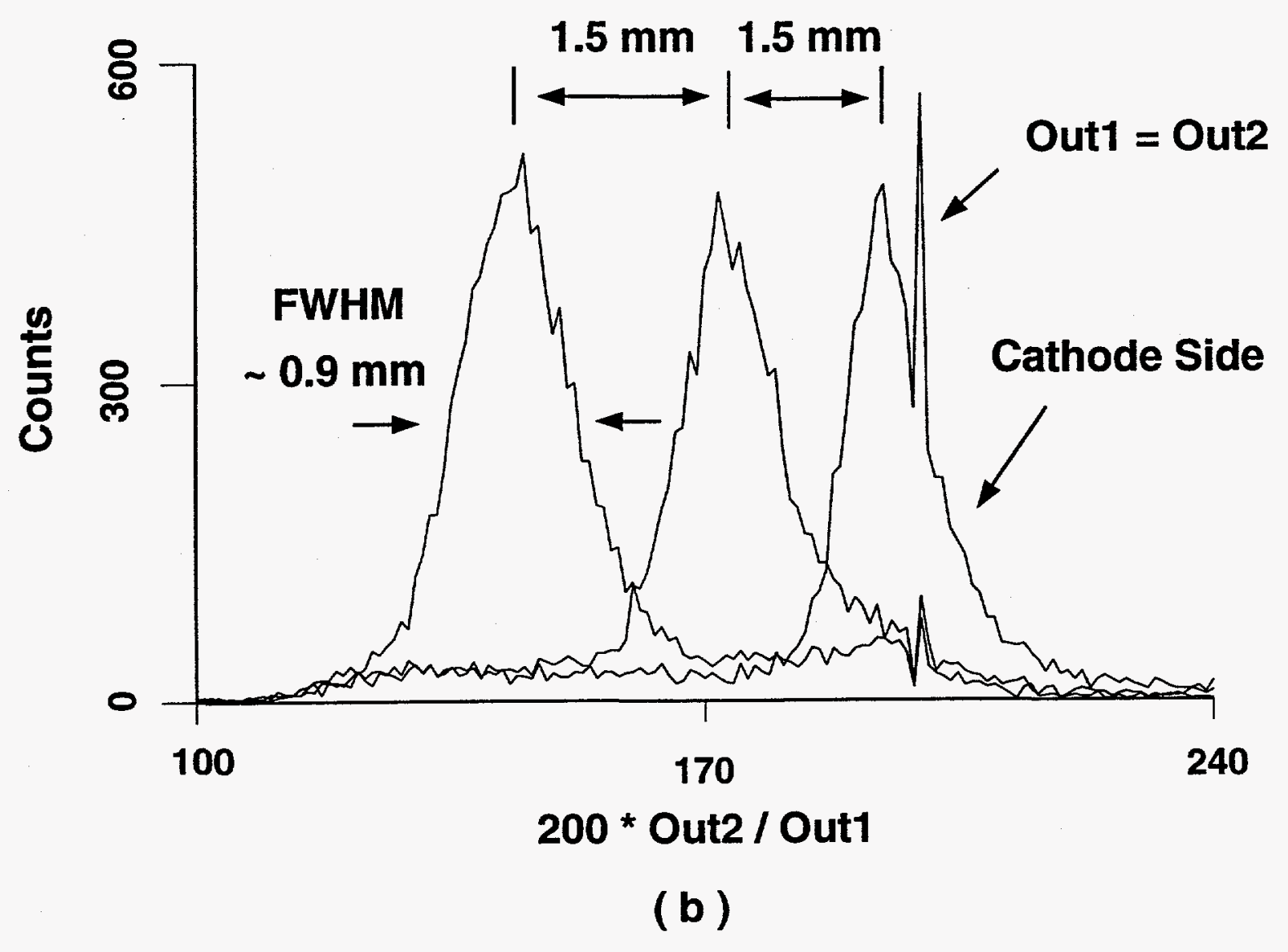

Fig. 4 


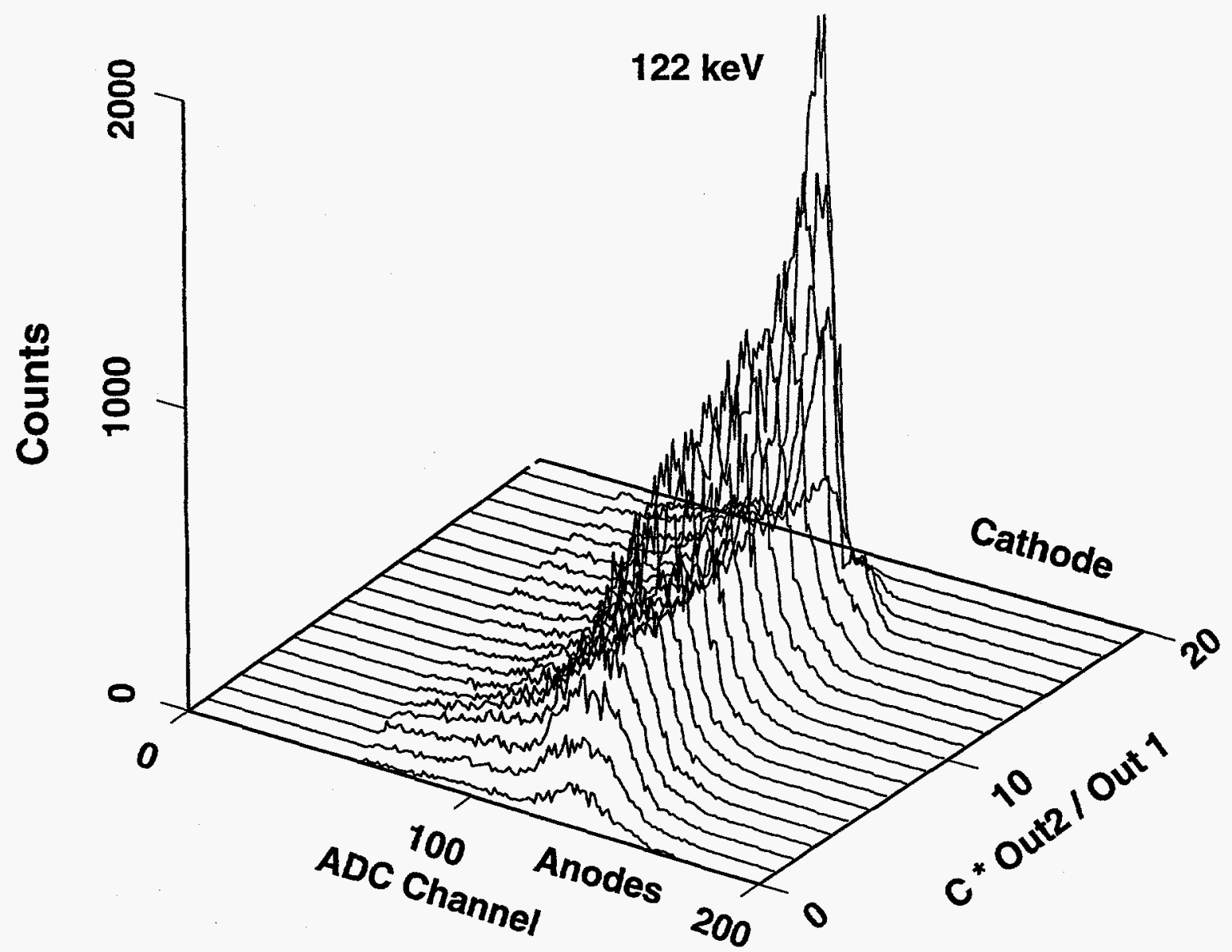

(a)

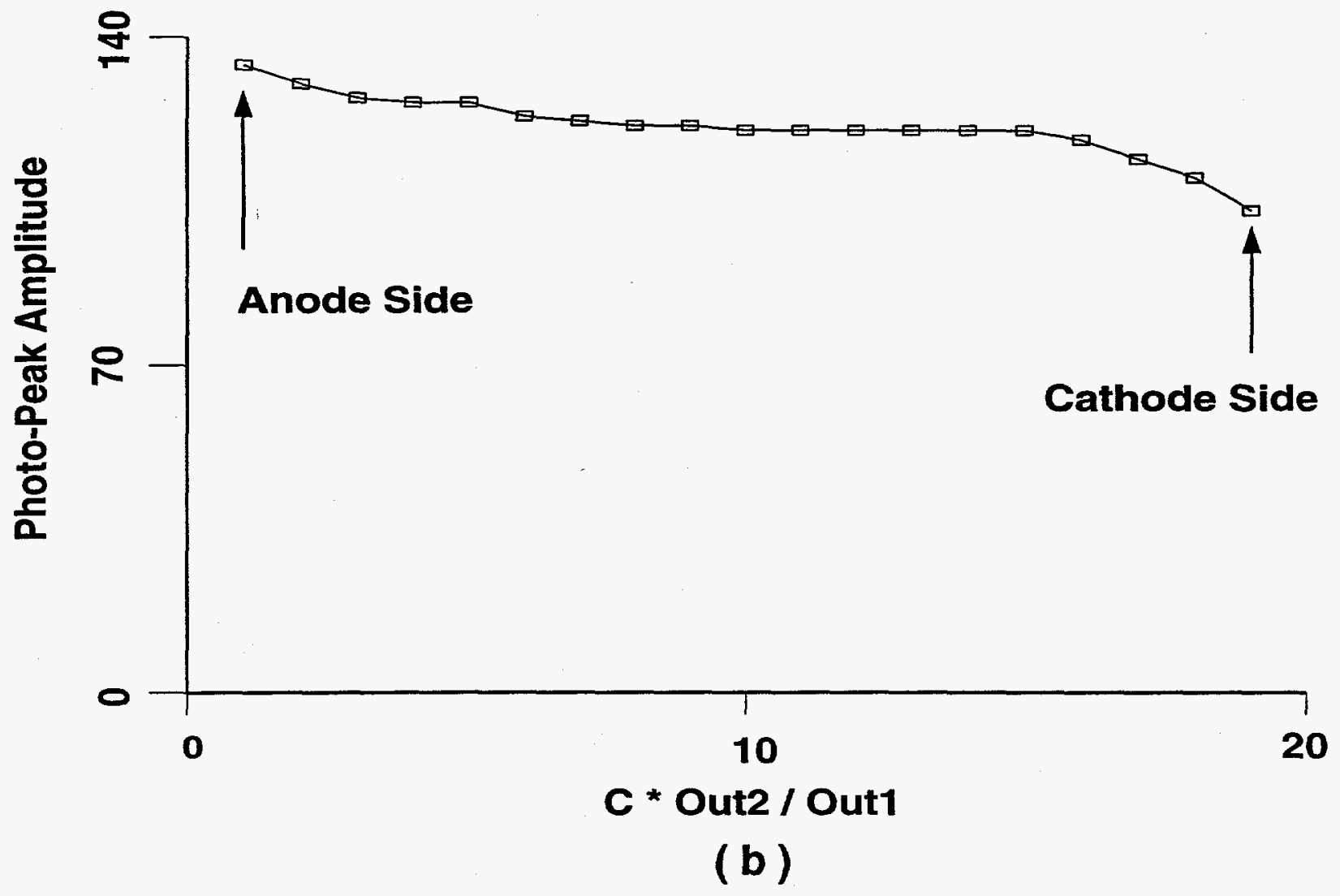

Fig. 5 


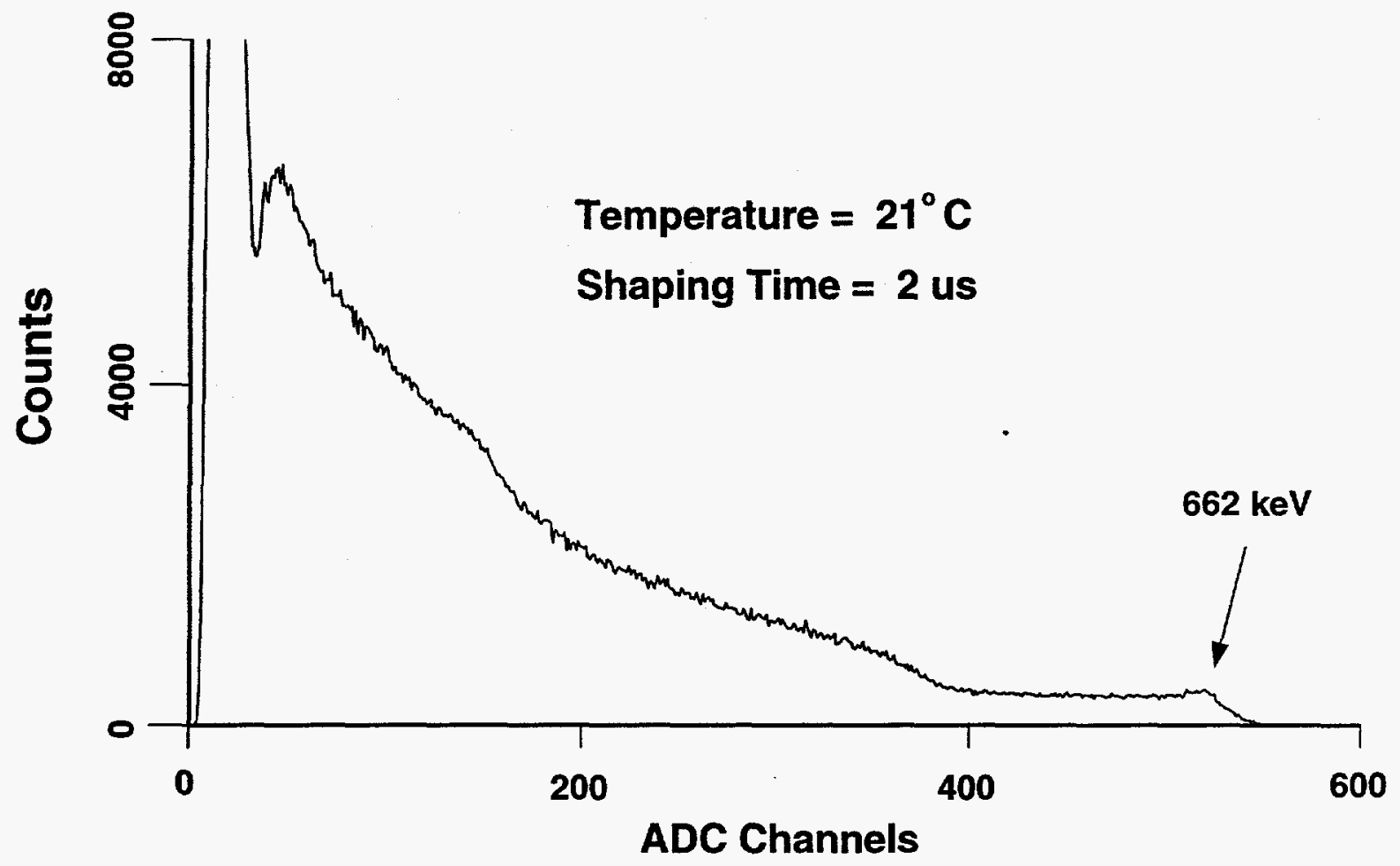

(a)

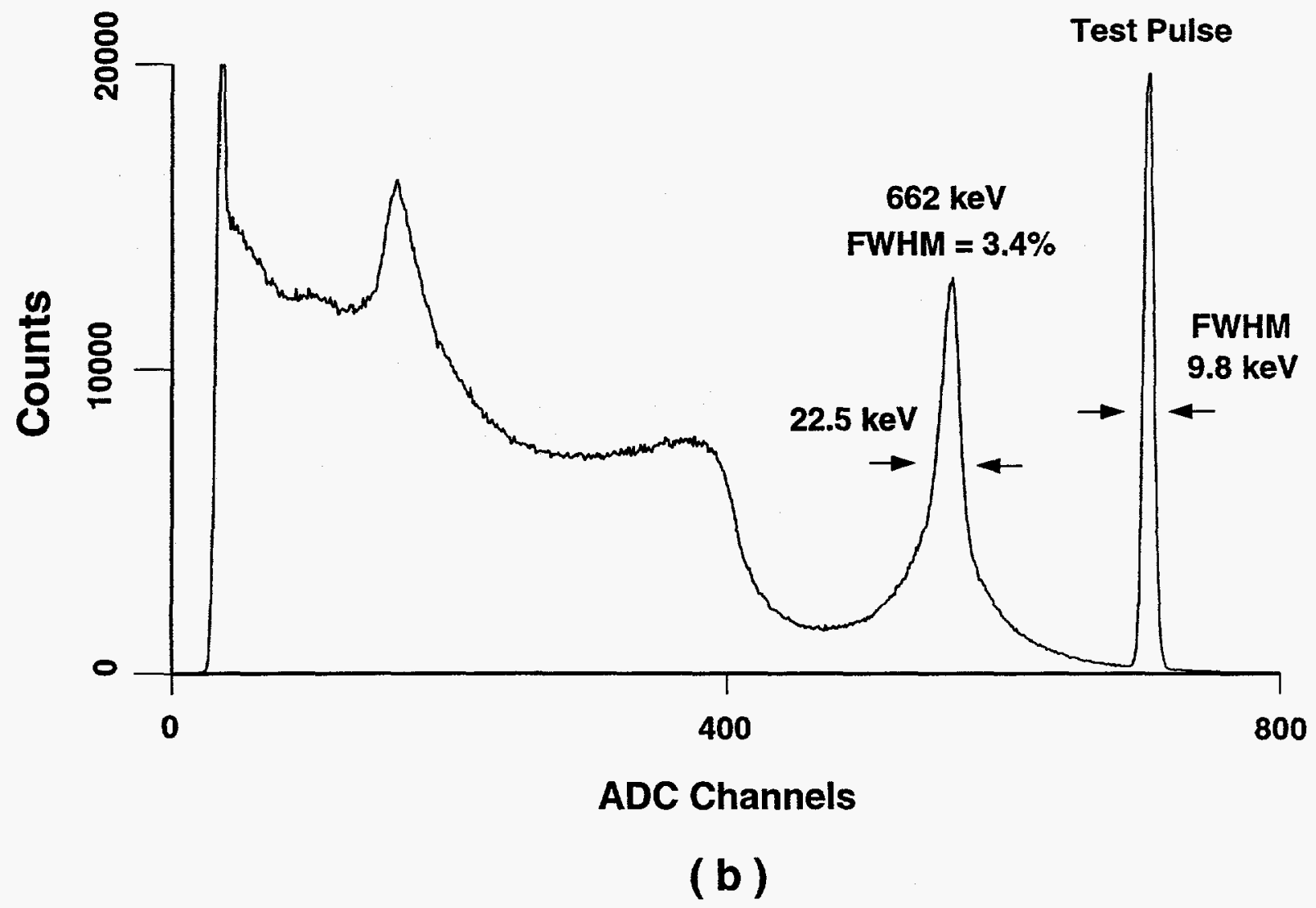

Fig. 6 


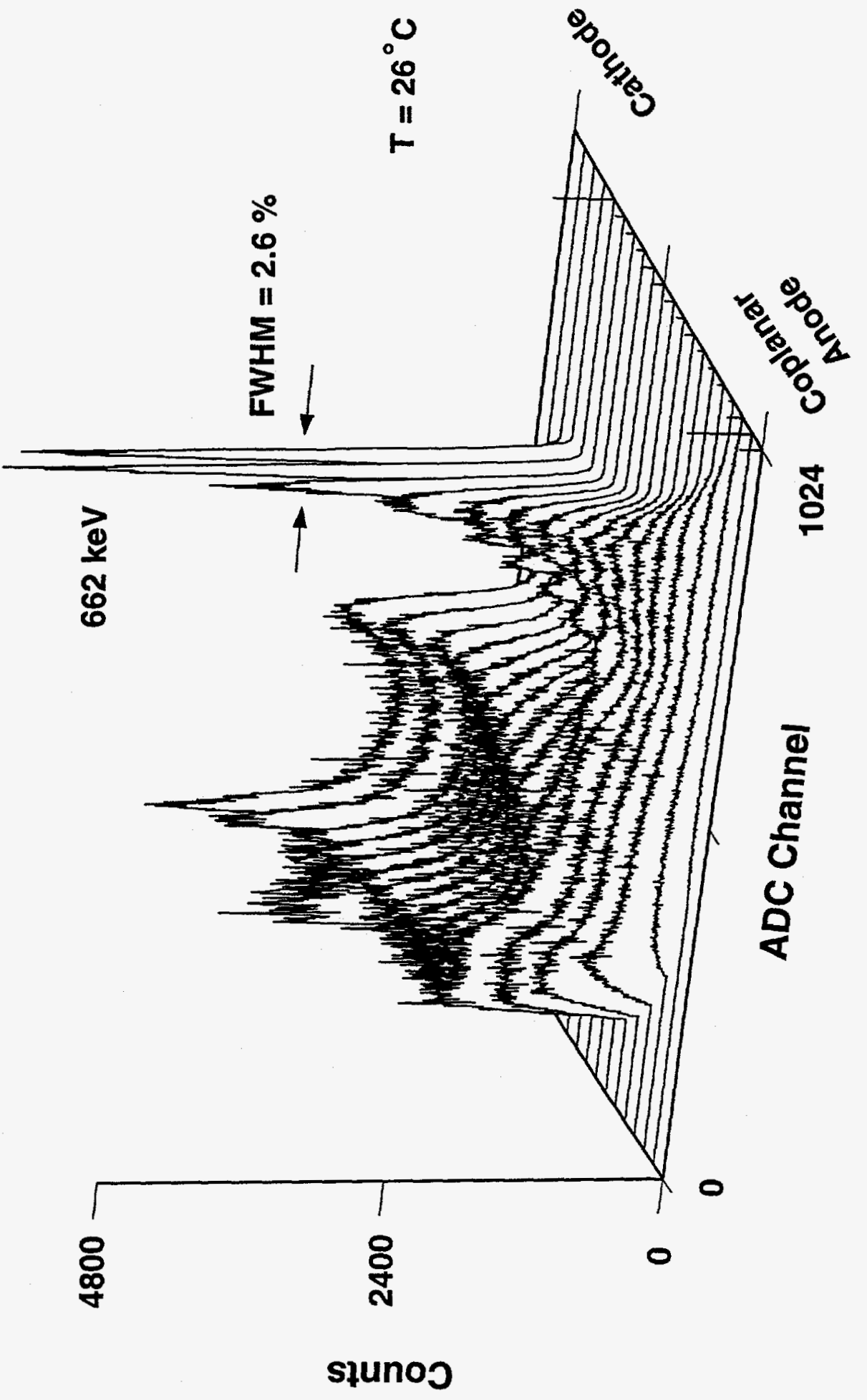

立 
APPENDIX III 


\section{Portable Directional Gamma-Ray Detector}

\section{Status Report}

November 1995

A portable detection system for gamma rays of energies between 90 and $300 \mathrm{keV}$ which is capable of source direction determination is under development. Several geometries have been postulated and a simple cubic arrangement (depicted in Figure 1) chosen for feasibility studies. In addition to source direction determination, a combination of three or more such detection systems can provide an estimation of the gamma-ray source location. Such a network is displayed in Figure 2.

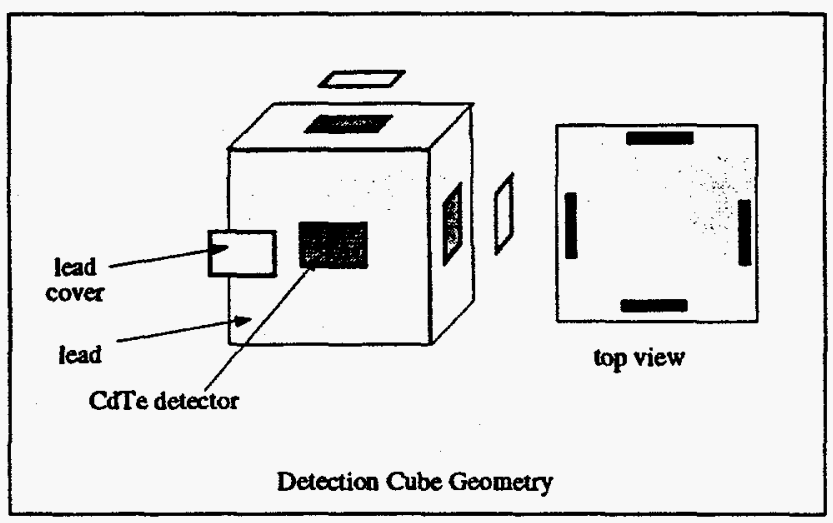

Figure 1: Detection Cube Design for Feasibility Study

The portability requirement for the detection system effectively eliminates the possibility of gamma spectroscopy on the multiple detectors required; however, a single channel analyzer (SCA) can be provided for each detector allowing detection of interactions within a specific energy range. The gamma-ray source energies are likely 


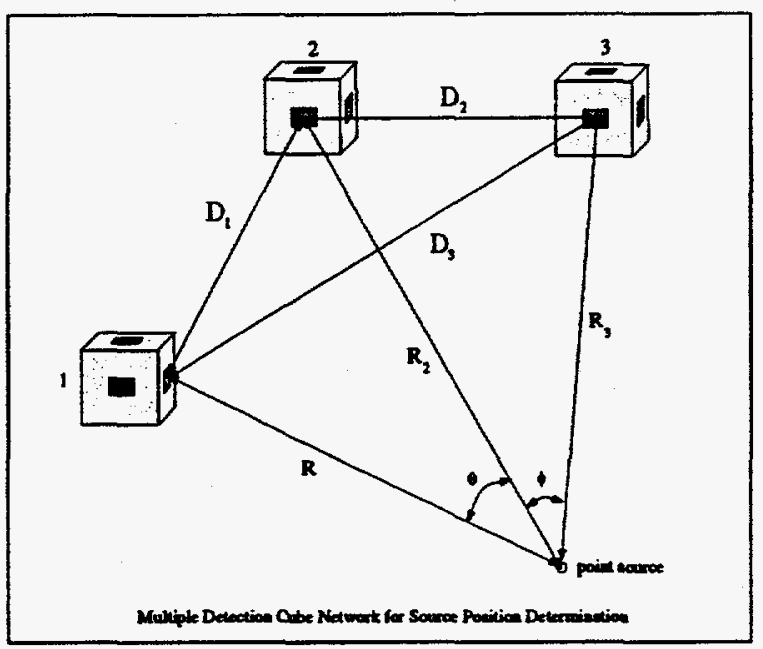

Figure 2: Application of Three Cubic Directional Detectors for Source Position Determination

to be distributed between $90 \mathrm{keV}$ and $300 \mathrm{keV}$ in an unknown fashion; therefore, the proposed detection method exploits the convenient $\mathrm{K}$-absorption edge at $88.21 \mathrm{keV}$ for x-ray emission in lead. That is, gamma rays with energies greater than 88.21 $\mathrm{keV}$ are capable (and probable) of exciting a lead atom and inducing the emission of a characteristic $x$ ray. The five most probable lead characteristic $x$-ray energies are $74.969,72.804,84.936,87.300$, and $84.450 \mathrm{keV}$, in order of abundance. Hence, by surrounding the detector with a thin layer of lead the detection of $x$ rays in the 74 to $85 \mathrm{keV}$ range should be proportional to the number of gamma rays incident on the detection cube face.

The detector material under investigation for this application is the room temperature semiconductor cadmium zinc telluride (CdZnTe or $\mathrm{CZT}$ ). The high stopping power of this material for lead $\mathrm{x}$ rays allows the use of very thin detectors $(1.2 \mathrm{~mm})$ on each face of the detection cube in Figure 1. An electron/gamma-ray Monte Carlo 
transport simulator has been used to determine the optimum thickness $(0.2 \mathrm{~mm})$ of the lead covering over each detector. Including the appropriate quantity of shielding between detectors, the minimum detection cube dimensions are $6.5 \mathrm{~cm} \times 6.5 \mathrm{~cm} \times$ $6.5 \mathrm{~cm}$, and the total mass of less than a kilogram. The bulk spatial requirements of the total detection system are expected to depend on the dimensions of the accompanying electronics. The immediate data processing could be executed on a microprocessor board about the size of one's hand, thereby leaving space for the high voltage supplies, amplifiers, and SCAs.

Current research efforts are directed toward obtaining simulated response functions for the detection cube, optimizing physical design parameters, and examining the minimum gamma-ray source intensity detection limits for the current design. 